\title{
Septic pulmonary emboli from mitral valve endocarditis in a patient with repaired tetralogy of fallot
}

Aniket S. Rali ${ }^{1}$, Arun lyer ${ }^{1}$, Claire Sullivan*2, James Strainic ${ }^{3}$, Brian Hoit ${ }^{2}$

${ }^{1}$ Department of Medicine, University Hospitals Case Medical Center, Case Western Reserve University, Cleveland Ohio, USA

${ }^{2}$ Harrington Heart and Vascular Institute, University Hospitals Case Medical Center, Case Western Reserve University, Cleveland Ohio, USA

${ }^{3}$ Rainbow Babies and Children's Hospital, Case Western Reserve University, Cleveland Ohio, USA

Received: May 10, 2016

DOI: $10.5430 /$ crim.v3n3p7
Accepted: May 25, 2016

Online Published: June 5, 2016

\begin{abstract}
A 37-year-old woman with a past medical history significant for congenital deafness and surgically repaired Tetralogy of Fallot presented with three day history of nausea, vomiting, fever, chills, dyspnea, and lower extremity weakness and physical examination notable for Janeway lesions. Peripheral blood and urine cultures were positive for methicillin sensitive Staphlococcus aureus. Transesophageal echocardiogram was consistent with mitral valve endocarditis. Computed tomography images of the chest, abdomen and pelvis demonstrated septic emboli to multiple organs including lungs, liver, spleen and kidneys. Saline contrast study was negative for a patent foramen ovale, or residual ventricular septal defect. Thus, effectively ruling out left to right intracardiac shunt as the cause of pulmonary septic emboli from mitral valve endocarditis. Moreover, cardiac MRI did not show any evidence of right sided endocarditis. Therefore, we believe the source of septic pulmonary emboli from mitral valve endocarditis to be through the bronchial arteries. The extent of septic emboli to various organs and the precise mechanism of pulmonary emboli from left sided endocarditis in a patient with surgically altered cardiac anatomy make this case unique.
\end{abstract}

Key Words: Tetralogy of fallot, Infective endocarditis, Septic emboli

\section{INTRODUCTION}

The annual incidence of infective endocarditis (IE) is estimated to be about 3 to 9 cases per 100,000 persons in industrialized countries. ${ }^{[1-7]}$ Individuals at greatest risk are those with prosthetic valves, intracardiac devices, unrepaired cyanotic congenital heart diseases or a prior history of IE. Other risk factors include chronic rheumatic heart disease $(<10 \%$ of cases in industrialized countries), hemodialysis, age-related degenerative valvular lesions ${ }^{[1,2,5]}$ and other co- morbidities such as HIV infection, diabetes and intravenous drug use. Infectious endocarditis in native as well as repaired ventricular septal defects (VSD) is a known and well documented phenomenon. ${ }^{[8]}$

This case report will discuss a young woman with repaired tetralogy of Fallot who presented with septic emboli to the lungs resulting from mitral valve endocarditis. Patient's postsurgical cardiac anatomy, extent of septic emboli to various organs and mechanism of pulmonary emboli from left sided

\footnotetext{
*Correspondence: Claire Sullivan, MD; Email: Claire.sullivan@uhhospitals.org; Address: 11100 Euclid Avenue, Mailstop Lakeside 5038, Cleveland, $\mathrm{OH} 44106$, USA.
} 
endocarditis make this case unique.
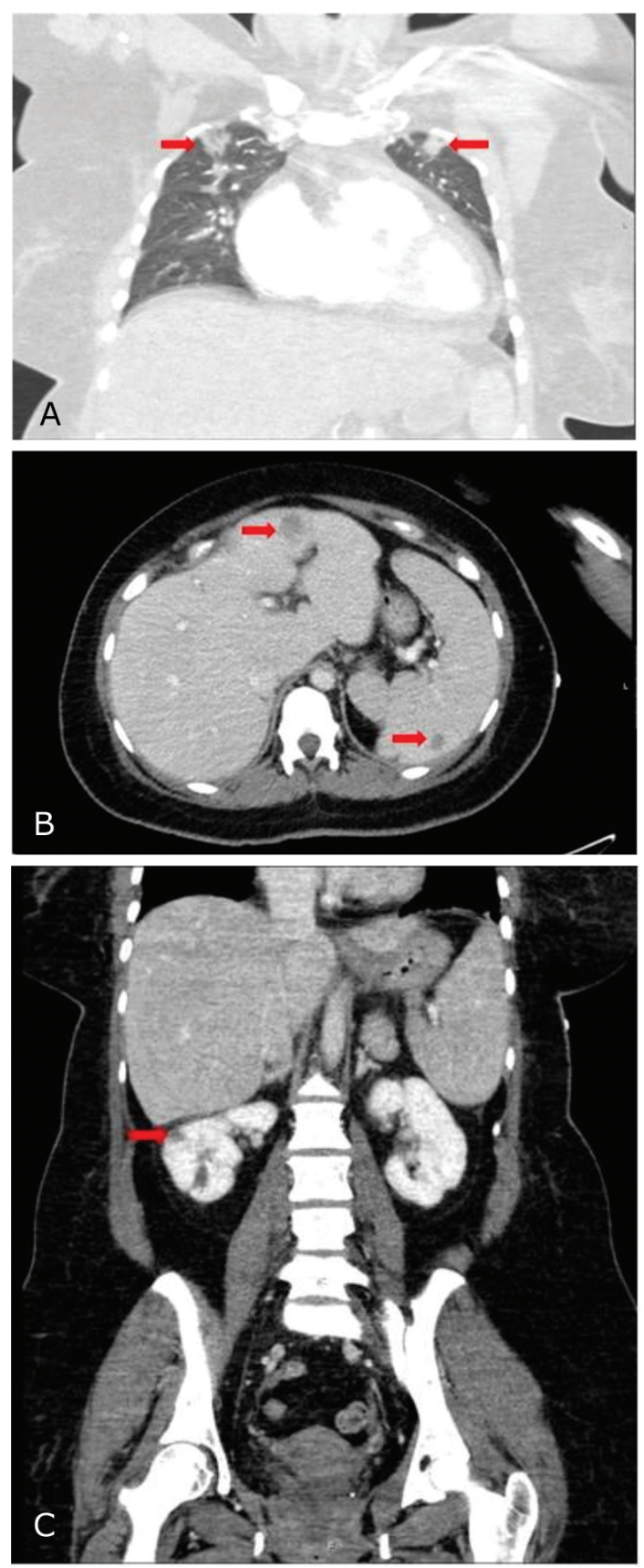

Figure 1. A. CT Chest showing septic pulmonary emboli; B. CT Abdomen-Pelvis showing hepatic and splenic emboli; C. CT Abdomen-Pelvis showing renal emboli

\section{CASe PResentation}

Ms. $\mathrm{T}$ is a 37-year-old woman with a past medical history significant for repaired tetralogy of Fallot (TOF) who initially presented to an outside hospital with a three day history of nausea, vomiting, fever, chills, shortness of breath and lower extremity weakness. Physical examination was notable for Janeway lesions. Peripheral blood and urine cultures were positive for methicillin sensitive staphylococcus aureus (MSSA). Laboratory: thrombocytopenia (platelet count of 52 $\mathrm{K})$, elevation in pro-inflamatory markers $(\mathrm{CRP}=373, \mathrm{ESR}=$ 38 and elevated procalcitonin) and mild elevation in bilirubin (total bilirubin of 3.4 and direct bilirubin of 2.7). Upon initial presentation, the patient met SIRS criteria and was admitted to the medical intensive care unit for further management of suspected severe sepsis. A transthoracic echocardiogram (TTE) did not show evidence of endocarditis. Computed tomographic (CT) scan of the chest, abdomen and pelvis was concerning for possible pulmonary (see Figure $1 \mathrm{~A}$ ), liver (see Figure 1 B) and renal emboli (see Figure 1 C). Continued lower extremity weakness raised the clinical suspicion for possible spinal cord compromise due to septic emboli but a non-contrast magnetic resonance imaging (MRI) of the thoracic and lumbar spine was negative for compression. She was then transferred to our facility for further care.

Blood cultures subsequently remained negative during the hospitalization in the setting of broad spectrum antibiotics. Repeat TTE again failed to show evidence of vegetation, although the tricuspid and pulmonic valves were difficult to visualize. Severe pulmonary regurgitation was noted on this study (see Figure 2). However, this was consistent with patient's prior TOF repair where her "pulmonary valve was excised along with hypertrophied septal and parietal bands". Saline contrast study was negative for a patent foramen ovale (PFO) and there was no evidence of residual ventricular septal defect (VSD). Transesophageal echocardiogram (TEE) revealed a $1.0 \mathrm{~cm} \times 0.40 \mathrm{~cm}$ mobile mass attached to the posterior annulus of the mitral valve (see Figures 3,4 ) consistent with infective endocarditis. Cardiac MRI was unremarkable. After seven weeks of intravenous antibiotics, follow up TEE showed resolution of mitral valve endocarditis.

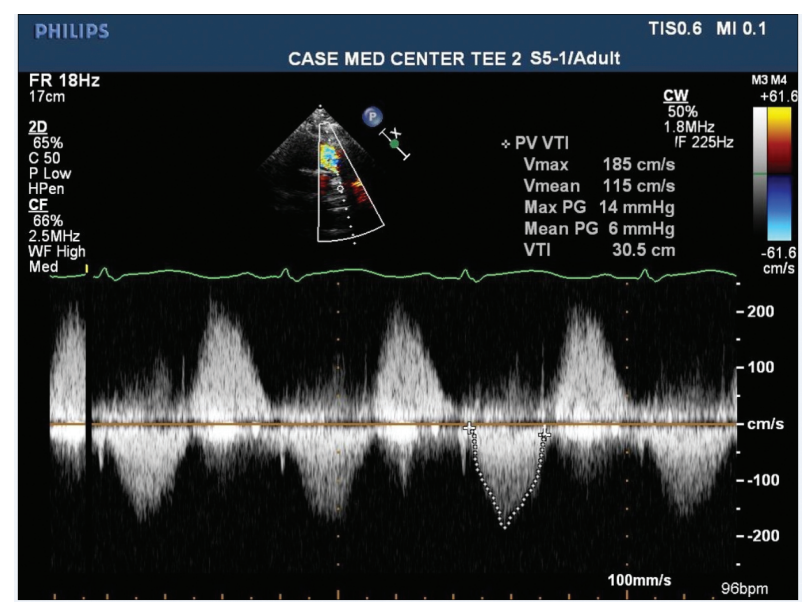

Figure 2. TTE showing severe pulmonic regurgitation 


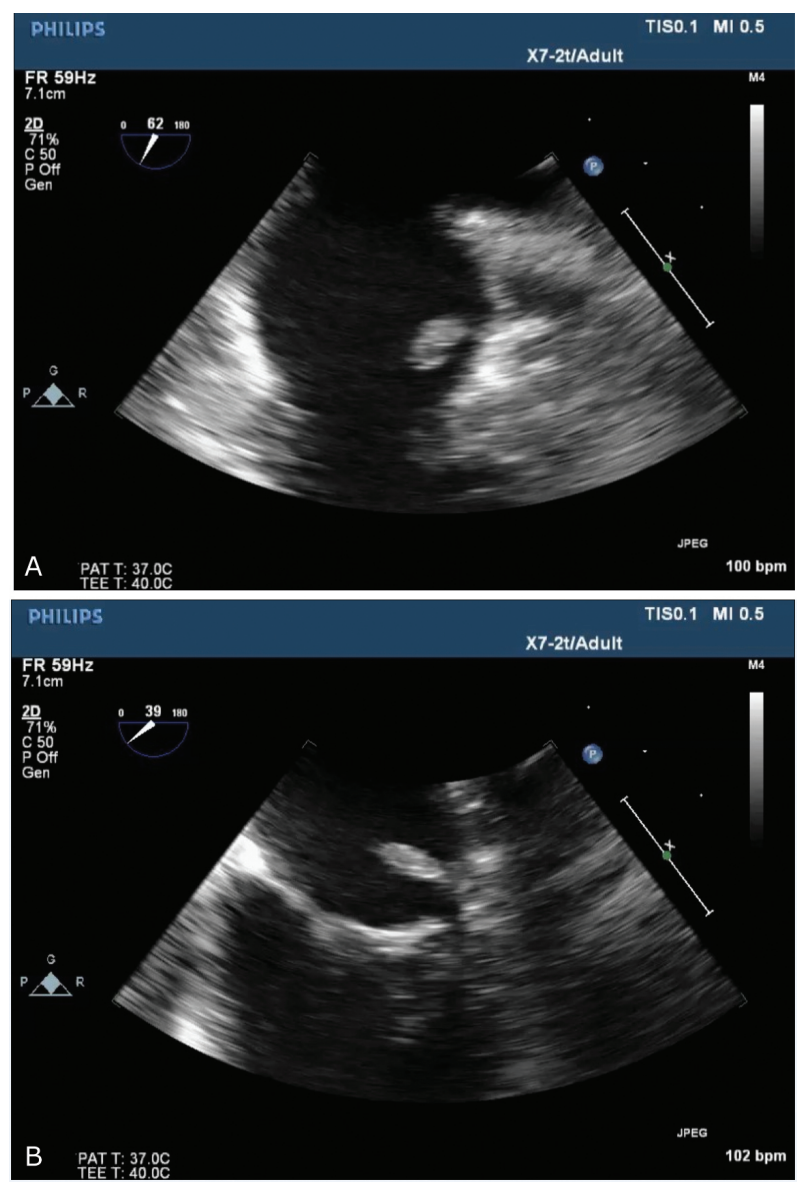

Figure 3. TEE demonstrating vegetation to posterior annulus of mitral valve

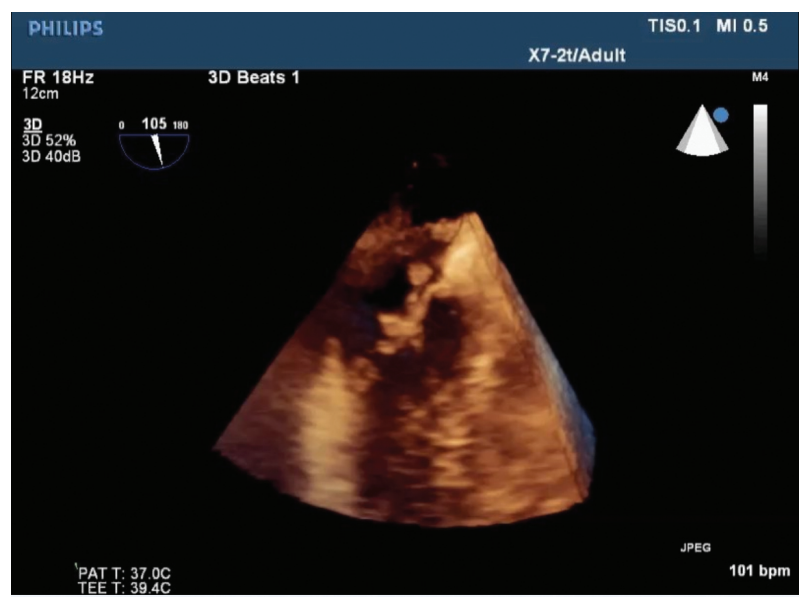

Figure 4. 3D TEE image again showing mitral valve posterior annulus vegetation

Throughout her hospitalization, the patient continued to report lower extremity weakness, now with foot numbness, and hence brain MRI was performed. MRI showed numerous scattered foci of acute to subacute infarction concerning for embolic phenomena (see Figure 5). Cerebral angiography Published by Sciedu Press imaging demonstrated a $1.7 \mathrm{~mm} \times 1.2 \mathrm{~mm}$ fusiform dilation of the parietal branch of the left middle cerebral artery concerning for mycotic aneurysm that was coiled (see Figure 6).

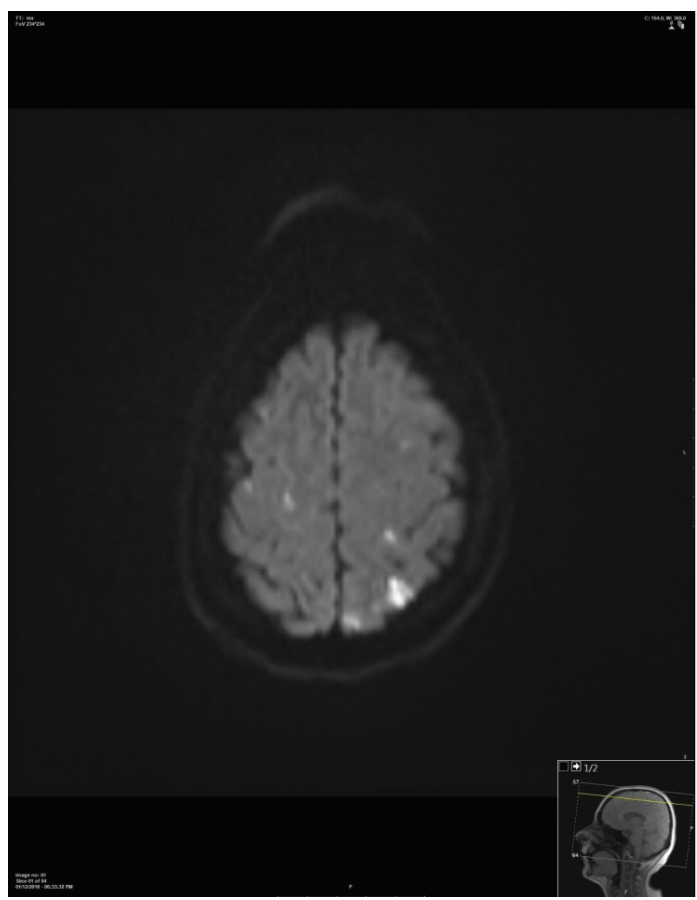

Figure 5. MRI Brain showing septic emboli

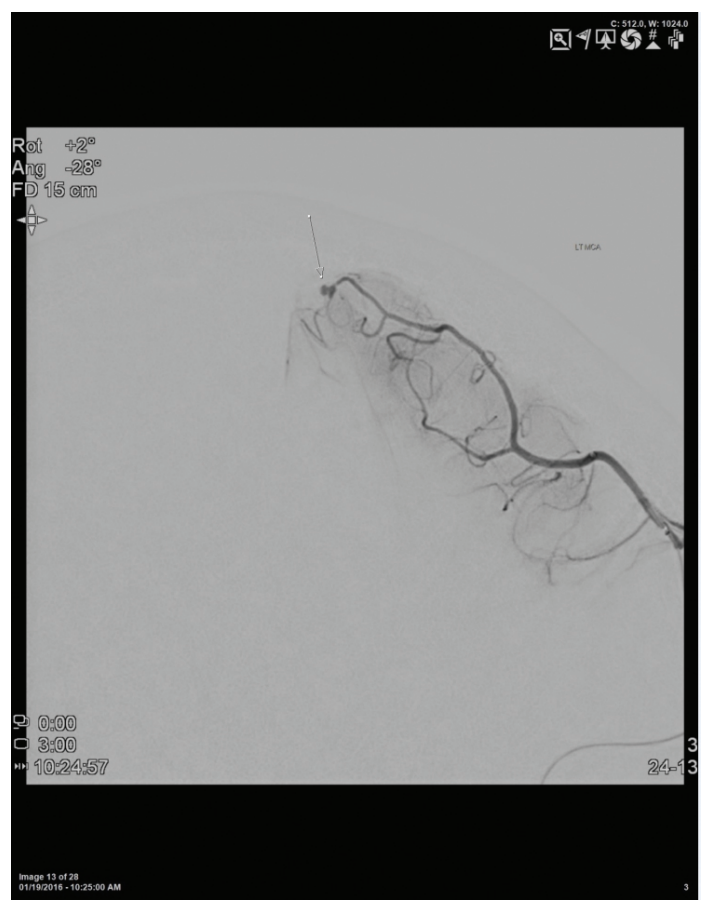

Figure 6. Mycotic Aneurysm of distal left middle cerebral artery

The patient also complained of severe pain in her left shoul- 
der throughout the hospitalization. An unsuccessful left shoulder aspiration was attempted at an outside hospital. MRI of the left shoulder showed a small glenohumeral joint effusion with evidence of inflammatory synovitis (see Figure 7).

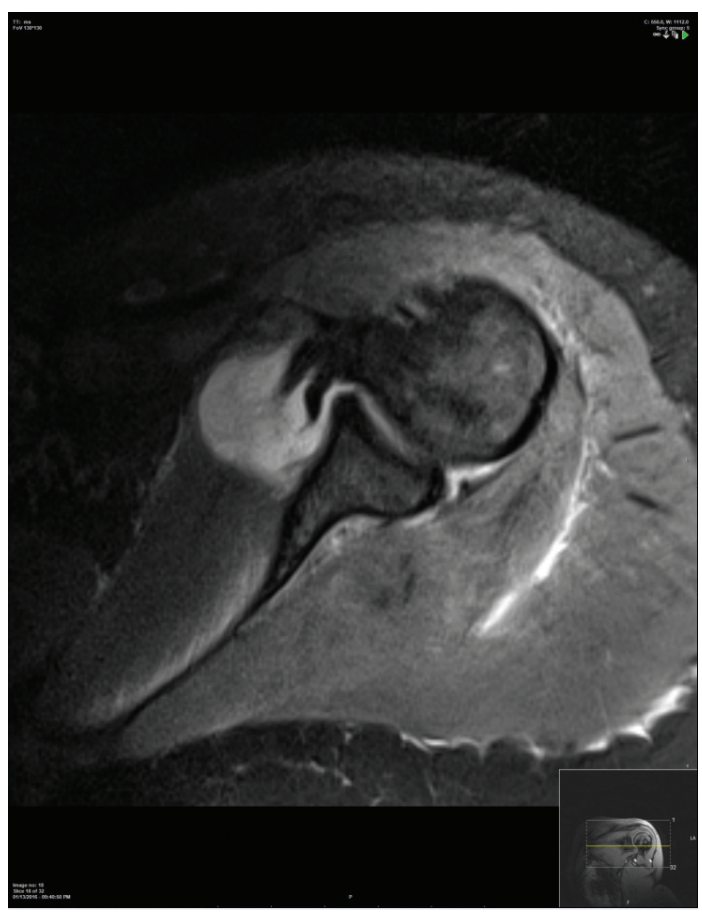

Figure 7. Shoulder MRI showing small effusion and edema of supraspinatus muscle

Patient was discharged from the hospital in a stable condition with plan for long term intravenous antibiotics.

\section{Discussion}

Ms. T presented with signs and symptoms of severe sepsis and was found to have infective endocarditis. Several aspects of this case make it unique namely the patient's post-surgical cardiac anatomy, potential sources of initial infection with MSSA, extent of septic emboli to various organs and most importantly pulmonary emboli from mitral valve endocarditis. Additionally, the mitral valve vegetation was atypical as it was less smudgy and more echo reflective than expected.

Our patient had a dental procedure performed two weeks prior to her initial presentation and that may have been the initial source of bacteremia. She did take prophylactic antibiotics. MSSA would be an atypical organism to cause bacteremia post dental procedures but certainly possible. It is unclear if MSSA in urine is a result of septic emboli to the kidney or a primary source of bacteremia.

Pulmonary valve regurgitation is a known complication of prior tetralogy of Fallot repair. Our patient had her native pulmonary valve excised during her surgical repair with residual severe pulmonary regurgitation. Such valve insufficiency can also occur in the setting of endocarditis. In our patient with obvious evidence of septic pulmonary emboli, the concern for right-sided endocarditis is high. In the absence of a pulmonary valve, the tricuspid valve becomes the prime suspect. However, there was no evidence of tricuspid valve vegetation on TEE. Furthermore, cardiac MRI did not show any signs of infected VSD patch. Alternatively, emboli could have originated from the infected mitral valve and traveled via a left to right shunt. However, in our patient there was no residual VSD or PFO.

Lungs receive dual blood supply from pulmonary as well as bronchial circulation. While this makes lung tissue more resistant to infarction, it also puts it at risk for emboli from both right and left sides of the heart. In our patient with known mitral valve endocarditis and extensive showering of emboli to liver, spleen, kidneys, brain and shoulder joint, we believe the mechanism of septic pulmonary emboli to be through the bronchial arteries. To the best of our knowledge, this is the first reported case of septic pulmonary emboli from left sided endocarditis in a patient with surgically repaired TOF. Patient did not require surgical repair of her mitral valve as no mitral valve vegetation was noted on TEE performed after seven weeks of intravenous antibiotics.

\section{REFERENCES}

[1] Correa de Sa DD, Tleyjeh IM, Anavekar NS, et al. Epidemiological trends of infective endocarditis: a population-based study in Olmsted County, Minnesota. Mayo Clin Proc. 2010; 85: 422 426. PMid:20435834 http://dx.doi.org/10.4065/mcp. 2009. 0585

[2] Duval X, Delahaye F, Alla F, et al. Temporal trends in infective endocarditis in the context of prophylaxis guideline modifications: three successive population-based surveys. J Am Coll Cardiol. 2012; 59: 1968-1976. PMid:22624837 http://dx.doi.org/10.1016/j.j acc. 2012.02.029

[3] Fedeli U, Schievano E, Buonfrate D, et al. Increasing incidence and mortality of infective endocarditis: a population-based study through a record-linkage system. BMC Infect Dis. 2011; 11: 4848. PMid:21345185 http://dx.doi.org/10.1186/1471-233 4-11-48

[4] Federspiel JJ, Stearns SC, Peppercorn AF, et al. Increasing US rates of endocarditis with Staphylococcus aureus: 1999-2008. Arch Intern Med. 2012; 172: 363-365. PMid:22371926 http://dx.doi.org /10.1001/archinternmed.2011.1027

[5] Sy RW, Kritharides L. Health care exposure and age in infective endocarditis: results of a contemporary population-based profile of 1536 patients in Australia. Eur Heart J. 2010; 31: 18901897. PMid:20453066 http://dx.doi.org/10.1093/eurhear $\mathrm{tj} / \mathrm{ehq110}$

[6] Murdoch DR, Corey GR, Hoen B, et al. Clinical presentation, eti- 
ology, and outcome of infective endocarditis in the 21st century: the International Collaboration on Endocarditis-Prospective Cohort Study. Arch Intern Med. 2009; 169: 463-473. PMid:19273776 http://dx.doi.org/10.1001/archinternmed.2008.603

[7] Selton-Suty C, Celard M, Le Moing V, et al. Preeminence of Staphylococcus aureus in infective endocarditis: a 1-year population-based survey. Clin Infect Dis. 2012; 54: 1230-1239. PMid:22492317 http://dx.doi.org/10.1093/cid/cis199

[8] Di Filippo S, Semiond B, Celard M, et al. Characteristics of infectious endocarditis in ventricular septal defects in children and adults Arch Mal Coeur Vaiss. 2004; 97(5): 507-14. PMid:15214556 